\title{
Beware of Pleural Thickening and Calcification: An Enlightenment from a Case of Tuberculous Pleurisy
}

This article was published in the following Dove Press journal:

Risk Management and Healthcare Policy

\author{
Yu Zhul,* \\ Yan-Hong $\mathrm{Gao}^{2}$,* \\ Jia-Ni Zou ${ }^{3}$ \\ Er-Ping $\mathrm{Xi}^{1}$ \\ 'Department of Thoracic Cardiovascular \\ Surgery, General Hospital of the Central \\ Theater Command of the People's \\ Liberation Army, Wuhan, 430070, \\ People's Republic of China; ${ }^{2}$ Department \\ of Ultrasound, General Hospital of the \\ Central Theater Command of the \\ People's Liberation Army, Wuhan, \\ 430070, People's Republic of China; \\ ${ }^{3}$ Department of Radiology, General \\ Hospital of the Central Theater \\ Command of the People's Liberation \\ Army, Wuhan, 430070, People's Republic \\ of China \\ *These authors contributed equally to \\ this work
}

\begin{abstract}
Tuberculous pleurisy, a type of extra-pulmonary tuberculosis, is the pleural inflammation caused by hypersensitive response of the pleural cavity stimulated by mycobacterium tuberculosis and its metabolites. If the patient does not receive timely and effective treatment and intervention, a large amount of fibrin will be deposited which will cause pleural thickening, adhesions, and even calcification. At present, clinical cases of typical pleural thickening and calcification have been rarely reported. In this paper, we will report a case of significant pleural thickening and calcification caused by tuberculous pleurisy; moreover, the patient earliest got tuberculous pleurisy about in 20 years ago and had received anti-tuberculous treatment at that time. The patient's typical crescent-shaped shadow created by pleural thickening and calcification is very rare in the domestic and international literature.
\end{abstract}

Keywords: tuberculosis, pleura, thickening, calcification

\section{Introduction}

Tuberculous pleurisy is a common clinical pleural inflammatory reaction mostly caused by the direct access of Mycobacterium tuberculosis in the pleura and is divided into dry pleurisy and exudative pleurisy; the former condition is often the early manifestation of the latter ${ }^{1-4}$. Even after receiving regular anti-TB treatment for tuberculous pleurisy, pleural thickening and calcification still occur in 10-72\% of patients. ${ }^{5}$ Pleural thickening and calcification may seriously affect the patient's respiratory function, work, and quality of life. Therefore, as for patient who suffered tuberculous pleurisy, the early diagnosis, early treatment, and early aspiration and drainage of pleural effusion are particularly important for the prevention of pleural thickening and calcification. This case report is expected to arouse clinicians' attention regarding the prevention and treatment of pleural thickening and calcification in tuberculous pleurisy and other thoracic diseases.

\section{Case Report}

A male patient, 60 years old, was admitted due to an intermittent cough that had been present for 2 weeks. According to the patient, he had suffered from tuberculous pleurisy about in 20 years ago, and at that time an assessment, made after 3 months' regular antiTB treatment, showed no explicit evidence indicated the tuberculosis remained. The physical examination revealed the following: the right lung activity was reduced, the
Correspondence: Er-Ping Xi; Jia-Ni Zou Command of the People's Liberation Army, 627\#, Wuluo Road, Wuchang District, Wuhan, Hubei, 430070, People's Republic of China

Tel +86-1387II 60246

Fax +86-27-50772388

Emailwo535I@I26.com;

dor_nizming@l63.com
Risk Management and Healthcare Policy 202 I:|4 |55|-I554 
mobility of the inferior boundary of the lung by percussion was $4-5 \mathrm{~cm}$, and the breathing sounds of the right lung were slightly weakened. Chest CT made after admission revealed a clear arcuate thickened calcification shadow in the right visceral pleura (Figure 1A) and obvious calcification lesions in the thickened pleura from the mediastinal window, with arcuate distribution and with a localized beaded shape (Figure 1B). Laboratory testing revealed a white blood cell count of $5.7 \times 10^{9} / \mathrm{L}$, an erythrocyte sedimentation rate (ESR) of $40 \mathrm{~mm} / \mathrm{h}$, and a C-reactive protein level of $8.08 \mathrm{mg} / \mathrm{L}$.

\section{Discussion}

Tuberculous pleurisy is a common clinical pleural inflammatory reaction mostly caused by the direct access of Mycobacterium tuberculosis in the pleura, which is type 4 tuberculosis. Based on the patient's medical history, clinical manifestations, tuberculosis culture and pathological findings of pleural needle biopsy tuberculous pleurisy can be diagnosed in general. ${ }^{6,7}$

Tuberculous pleurisy can be divided into dry pleurisy and exudative pleurisy, and the former condition is often the early manifestation of the latter. In the early stage of tuberculous pleurisy, pleural congestion and edema can be observed, and the capillary osmotic pressure is increased, which will cause a large amount of exudate to enter the chest. The pleural exudate contains a large amount of fibrin, which can accumulate in the pleura and clog the lymphatic vessels, thus hindering the absorption of the exudate and forming the pleural effusion, causing pleural wrapping and adhesions and eventually leading to pleural thickening and calcification. Extensive pleural thickening and calcification can cause severe restrictive ventilation disorders, ${ }^{8}$ which manifest as restricted breathing, chest

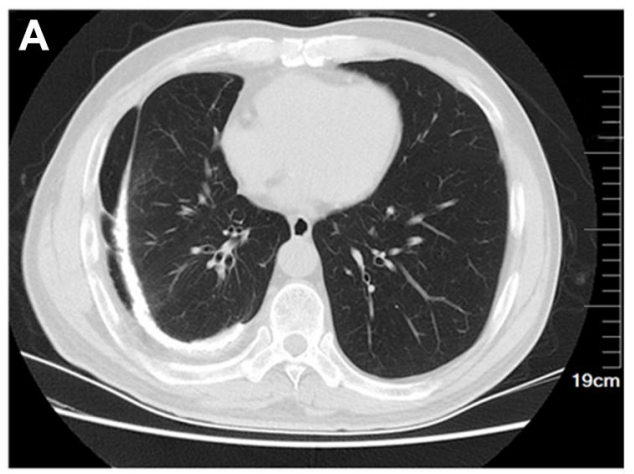

pain, and intercostal space narrowing. In addition, if a patient has both extensive pleural calcification and lung cancer in the affected thoracic cavity. Then this patient may lose the opportunity to receive surgical treatment because there is no operative approach.

The occurrence of pleural thickening and calcification in tuberculous pleurisy is closely related to the treatment time, the cell number, the protein content, and the timely extraction of the pleural effusion. Even after regular clinical anti-TB treatment, the incidence still ranges from $10 \%$ to $72 \%{ }^{5}$ Therefore, when meeting the tuberculous pleurisy, in addition to anti-TB treatment, the prevention and treatment of pleural thickening and calcification also deserve to be concerned. Pleural thickening adhesions and calcification can be prevented by the combination of intra-thoracic injection of urokinase and sodium hyaluronate, mycobacterium-assisted anti-TB aspiration, drainage of pleural effusions, and hormone therapy to promote the absorption of pleural effusion. ${ }^{9-12}$ For patients with pleural thickening and calcification, the total adenosine deaminase in the body is significantly increased, suggesting that the mechanism of pleural thickening may be related to the cellular immune activity. ${ }^{13}$ Decortication of pleural fiberboard or thoracoplasty is feasible when the pleural thickness is approximately $0.5 \mathrm{~cm}$, which can significantly reduce adhesions in the visceral pleura and lung tissue. ${ }^{14}$ Of course, there are still some objections to the application of glucocorticoids in the treatment of tuberculous pleurisy. Although this treatment strategy can significantly reduce the number of pleural effusions in a short amount of time, significantly more adverse reactions have occurred in patients receiving glucocorticoids treatment than in those not using glucocorticoids, and there is no conclusive evidence regarding their effect on pleural thickening; Thus, their clinical application remains to be further explored. ${ }^{15}$

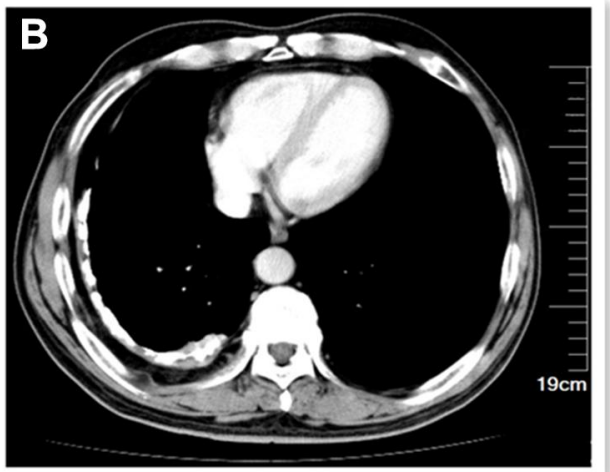

Figure I Chest CT examination of the lung window revealed an obvious clear arcuate thickened calcification shadow in the right pleura (A) and obvious calcification lesions in the thickened pleura from the mediastinal window, with arcuate distribution and with a localized beaded shape (B). 
At present, the specific mechanism of pleural thickening in patients with tuberculous pleurisy is still unclear, and there are no definite preventive and curative measures used in clinical practice currently. Therefore, clinicians should actively and flexibly adjust the treatment plan during the management of patients with tuberculous pleurisy to prevent the occurrence of pleural thickening.

\section{Ethics}

The authors are accountable for all aspects of the work in ensuring that questions related to the accuracy or integrity of any part of the work are appropriately investigated and resolved. This study complies with the guidelines for human studies and is in accordance with the Declaration of Helsinki. The ethics Review Committee of General Hospital of Central Theater Command of the People's Liberation Army approved the use of clinical data of this patient in this study (2020 Provincial Key R\&D Plan-279).

\section{Informed Consent for Publication}

Written informed consent was obtained from the patient for the publication. The patient provided written informed consent to participate in this study.

\section{Acknowledgment}

These authors contributed equally to this work and should be considered as co-first authors: Yan-Hong Gao and Yu Zhu.

\section{Funding}

This paper is supported by grants from, CHINA [No. 2020BCB059].Primary Research \& Development Plan of Hubei Province.

\section{Disclosure}

The authors declare that they have no conflicts of interest for this work.

\section{References}

1. Wang W, Zhou Q, Zhai K, et al. Diagnostic accuracy of interleukin 27 for tuberculous pleural effusion: two prospective studies and one meta-analysis. Thorax. 2018;73(3):240-247. doi:10.1136/thoraxjnl2016-209718
2. Zhang Q, Zhou C. Comparison of laboratory testing methods for the diagnosis of tuberculous pleurisy in China. Sci Rep. 2017;7(1):4549. doi:10.1038/s41598-017-04872-6

3. Tong X, Lu H, Yu M, Wang G, Han C, Cao Y. Diagnostic value of interferon- $\gamma$-induced protein of $10 \mathrm{kDa}$ for tuberculous pleurisy: a meta-analysis. Clin Chim Acta. 2017;471:143-149.

4. Xu HY, Zhang DQ, Ye JR, et al. Diagnostic performance of T-SPOT. TB on peripheral blood in combination with adenosine deaminase on pleural fluid for the diagnosis of tuberculous pleurisy within different age group. Zhonghua Yi Xue Za Zhi. 2017;97(24):1862-1866. doi:10.3760/cma.j.issn.0376-2491.2017.24.004

5. Gopi A, Madhavan SM, Sharma SK, Sahn SA. Diagnosis and treatment of tuberculous pleural effusion in 2006 [J]. Chest. 2007;131 (3):880-889. doi:10.1378/chest.06-2063

6. Mollo B, Jouveshomme S, Philippart F, Pilmis B. Biological markers in the diagnosis of tuberculous pleural effusion. Ann Biol Clin. 2017;75(1):19-27.

7. Amer S, Hefnawy AE, Wahab NA, Okasha H, Baz A. Evaluation of different laboratory methods for rapid diagnosis of tuberculous pleurisy. Int $J$ Mycobacteriol. 2016;5(4):437-445. doi:10.1016/j. ijmyco.2016.07.001

8. Behrsin RF, Junior CT, Cardoso GP, Barillo JL, de Souza JB, de Araújo EG. Combined evaluation of adenosine deaminase level and histopathological findings from pleural biopsy with Cope's needle for the diagnosis of tuberculous pleurisy. Int J Clin Exp Pathol. 2015;8 (6):7239-7246.

9. Ding D, Deng Q, Zhang H. The study of intrapleural urokinase in the prevention of pleural thickening and loculated effusions by tuberculous pleurisy. Zhonghua Jie He He Hu Xi Za Zhi. 2001;24(1):32-34.

10. Cao GQ, Li L, Wang YB, Shi ZZ, Fan DY, Chen HY. Treatment of free-flowing tuberculous pleurisy with intrapleural urokinase. Int J Tuberc Lung Dis. 2015;19(11):1395-1400. doi:10.5588/ ijtld. 15.0128

11. Liang $\mathrm{Y}, \mathrm{Wu} \mathrm{X}$, Zhang $\mathrm{J}$, et al. Immunogenicity and therapeutic effects of $\mathrm{Ag} 85 \mathrm{~A} / \mathrm{B}$ chimeric DNA vaccine in mice infected with Mycobacterium tuberculosis. FEMS Immunol Med Microbiol. 2012;66(3):419-426. doi:10.1111/1574-695X.12008

12. Zhou A, Guo L, Tang L. Effect of an intrathoracic injection of sodium hyaluronic acid on the prevention of pleural thickening in excess fluid of tuberculous thoracic cavity. Clin Exp Pharmacol Physiol. 2003;30(3):203-205. doi:10.1046/j.1440-1681.2003. 03804.x

13. Uskul B, Turker H, Ulman C, et al. The relation of the pleural thickening in tuberculosis pleurisy with the activity of adenosine deaminase. Monaldi Arch Chest Dis. 2005;63(2):101-107. doi:10.4081/monaldi.2005.646

14. Krasnov D, Krasnov V, Skvortsov D, Felker I. Thoracoplasty for tuberculosis in the twenty-first century. Thorac Surg Clin. 2017;27 (2):99-111. doi:10.1016/j.thorsurg.2017.01.003

15. Ryan H, Yoo J, Darsini P. Corticosteroids for tuberculous pleurisy. Cochrane Database Syst Rev. 2017;3:CD001876. doi:10.1002/ 14651858.CD001876.pub3 


\section{Publish your work in this journal}

Risk Management and Healthcare Policy is an international, peerreviewed, open access journal focusing on all aspects of public health, policy, and preventative measures to promote good health and improve morbidity and mortality in the population. The journal welcomes submitted papers covering original research, basic science, clinical \& epidemiological studies, reviews and evaluations, guidelines, expert opinion and commentary, case reports and extended reports. The manuscript management system is completely online and includes a very quick and fair peer-review system, which is all easy to use. Visit http://www.dovepress.com/testimonials.php to read real quotes from published authors. 